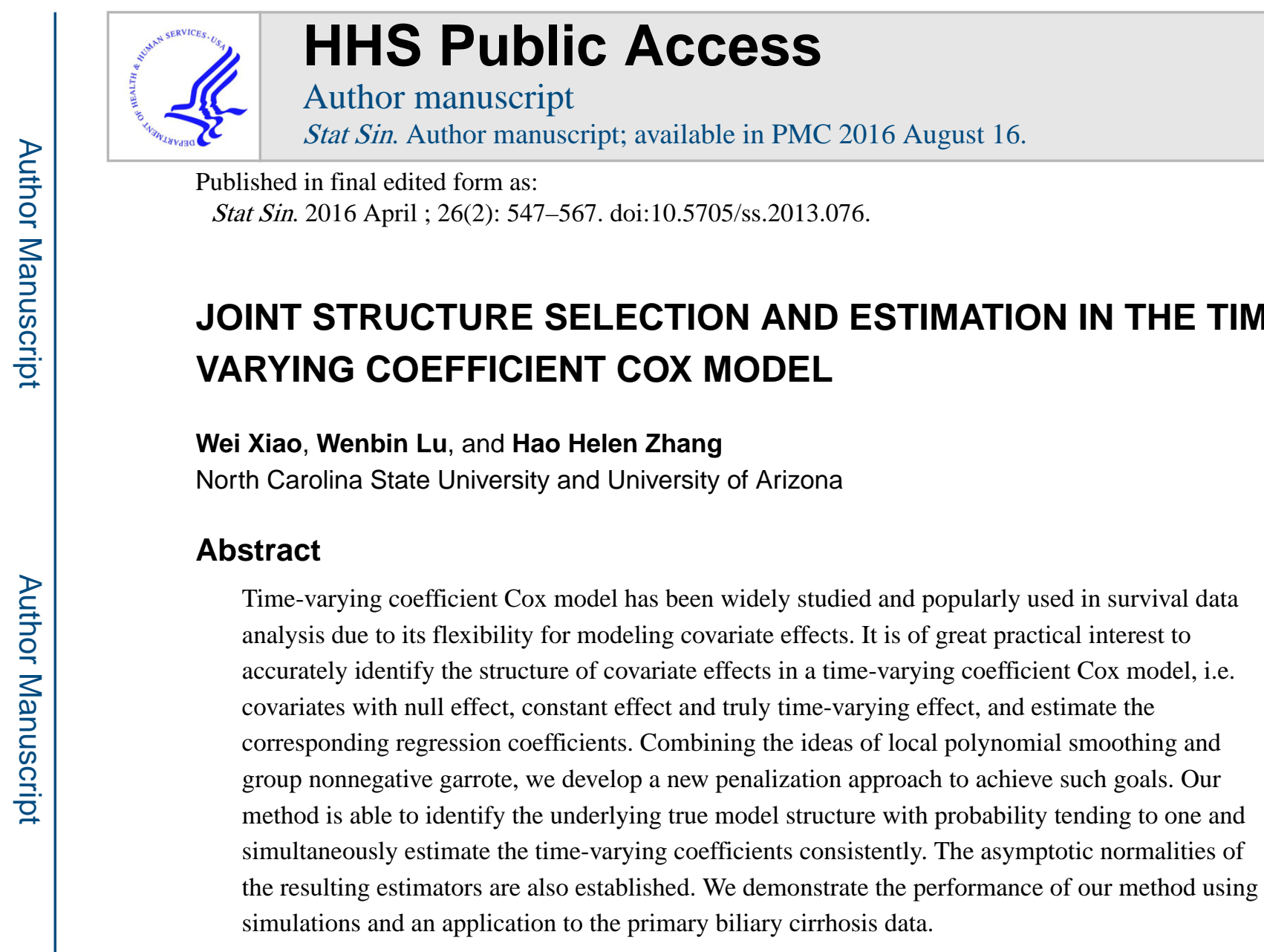

\title{
Key words and phrases
}

Group nonnegative garrote; local polynomial smoothing; model selection; time-varying coefficient Cox model

\section{Introduction}

Cox proportional hazards model (Cox, 1972) has become the most popularly used semiparametric model in survival analysis due to its nice hazard interpretation and easy estimation based on partial likelihood principle with elegant counting process-based martingale theory (Andersen and Gill, 1982). However, one main limitation of the standard Cox model is to assume that the hazard ratios stay constant over time, i.e. covariate effects are time-invariant, which may be unrealistic in practical applications. Many alternatives have been proposed to relax the proportional hazards assumption. Among them, time-varying coefficient Cox model is a natural extension of the standard Cox model by allowing temporal effects of covariates, and has been widely studied in the literature (e.g. Zucker and Karr, 1990; Cai and Sun, 2003; Tian et al., 2005).

Department of Statistics, North Carolina State University, Raleigh, NC 27695-8203, U.S.A. (wxiao@ncsu.edu) Department of Statistics, North Carolina State University, Raleigh, NC 27695-8203, U.S.A. (lu@ stat.ncsu.edu) Department of Mathematics and Statistics Interdisciplinary Program, University of Arizona, Tucson, AZ 85721-0089, U.S.A. (hzhang@math.arizona.edu) 
An important issue in fitting a time-varying coefficient Cox model is to identify the true model structure, i.e. to distinguish covariates with null effect, constant effect or truly timevarying effect, since it can help to build a more accurate risk model. First, by identifying covariates with null effect and excluding them from the model, it can greatly reduce the dimension of model, which is important for building a parsimonious and reliable risk model especially when $p$ is large and many of the covariates have no effects on the survival time. Second, by distinguishing covariates with constant effect and truly time-varying effect, we can build a simpler and easier-interpretable semiparametric model comparing with a pure nonparametric model with time-varying coefficients, which will be highly appreciated by empirical investigators since they generally prefer a flexible but easy-interpretable model for data analysis. For example, Yu and Lin (2010) considered a data from the western Kenya parasitemia study, and found that exposure to mosquito bites (BITE), age and gender have constant effects on time to onset of parasitemia, while baseline parasitemia density (BPD) has time-varying effect. Motivated by the Western Kenya data, they considered a semiparametric time-varying coefficient model for better risk prediction. See Zhang et al. (2002); Fan and Huang (2005); Ahmad et al. (2005); Wang et al. (2009) for more demonstration of the benefits of semiparametric varying-coefficient models comparing with nonparametric varying-coefficient models.

Model selection has been extensively studied in the past few decades. Traditional model selection techniques, such as best-subset selection, coupled with $C_{p}$ (Mallows, 1973), AIC (Akaike, 1973) and BIC (Schwarz, 1978), separate model selection and model estimation steps and are generally unstable due to the their inherent discreteness (Breiman, 1995) and stochastic errors (Fan and Li, 2001). They also lack of asymptotic selection consistency, which is a desirable asymptotic property to possess. More importantly, they are not computational feasible for data set with moderate to large dimensions as their computation times increase exponentially with the dimension. To overcome these difficulties, various penalization methods have been introduced, for example, nonnegative garrote (Breiman, 1995), LASSO (Tibshirani, 1996, 1997), SCAD (Fan and Li, 2001, 2002) and adaptive LASSO (Zou, 2006; Zhang and Lu, 2007). These methods provide competing performance for simultaneously selecting important variables and estimating their effects. However, most existing penalization methods focus on variable selection for simple linear regression models. Less has been studied for model structure selection, for example, the identification of linear/nonlinear structure in partially linear regression models or time-invariant/timevarying coefficients in regression models with time-varying coefficients. Recently, Zhang et al. (2011) proposed a novel penalization approach in the frame of smoothing spline ANOVA for automatically discovering covariates with null effect, linear effect and nonlinear effect in a partially linear model. For censored data, Yan and Huang (2012) proposed an adaptive group LASSO (AGLASSO) method based on a penalized B-spline approach for model structure selection in a time-varying coefficient Cox model. Specifically, time-varying coefficients are expanded with a set of B-spline basis functions and an adaptive group lasso penalty is used to select between time-independent and time-dependent covariate effects.

In this paper, we propose an alternative method for automatic model structure selection and coefficient estimation in a time-varying coefficient Cox model by coupling the kernelweighted partial likelihood estimation (Cai and Sun, 2003; Tian et al., 2005) with the group 
nonnegative garrote penalty. There are three major motivations for developing this new approach based on local kernel methods. First, in contrast with the spline method proposed in Yan and Huang (2012), our method is able to better capture some local features of timevarying coefficient functions, which are otherwise hard to be captured by the spline method. Second, by using the local kernel estimation, it enables us to rigorously study the asymptotic properties of the proposed estimators for both constant and time-varying coefficients, such as model selection consistency and asymptotic normality, and hence justify the validity of the methods from theoretical perspectives. None of these properties have been established for existing approaches like Yan and Huang (2012). Third, the proposed method provides an automatic and effective way to conduct structure selection for time-varying coefficient Cox model, which can deal with relative large dimension in contrast with all existing methods based on hypothesis testing, such as those studied in Huang et al. (2002), Fan and Huang (2005), Tian et al. (2005) and Liu et al. (2010). The remainder of the paper is organized as follows. Our proposed kernel group nonnegative garrote (KGNG) method and its variant (KGNG2) are introduced in Section 2. Asymptotic properties of KGNG and KGNG2 estimators are presented in Section 3. Section 4 is devoted to numerical studies, including simulations and an application to the primary biliary cirrhosis data. All the technical proofs are relegated to an online supplementary appendix available at http://www.stat.sinica.edu.tw/ statistica.

\section{Structure Selection with Kernel Group Nonnegative Garrote}

\subsection{Methods}

Consider a random sample of $n$ individuals. Let $T_{i}$ be the failure time, $C_{i}$ be the censoring time, and $\boldsymbol{Z}_{i}$ be a $p$-vector of covariates for subject $i$. Conditional on $\boldsymbol{Z}_{i}, T_{i}$ and $C_{i}$ are assumed independent. Define $\tilde{T}_{i}=\min \left(T_{i}, C_{i}\right)$ and $\Delta_{i}=\mathbb{1}\left(T_{i} \leq C_{i}\right)$. The data consist of the triplets $\left(\tilde{T}_{i}, \boldsymbol{Z}_{i}, \Delta_{i}\right), i=1, \ldots, n$. The time-varying coefficient Cox model assumes the following form

$$
\alpha\left(t \mid \boldsymbol{Z}_{i}\right)=\alpha_{0}(t) e^{\boldsymbol{\beta}_{0}^{\top}(t) \boldsymbol{Z}_{i}},
$$

where $a\left(\cdot \mathbb{Z}_{i}\right)$ is the conditional hazard function given covariates, $a_{0}(\cdot)$ is a completely unspecified baseline hazard function, and $\beta_{0}(t)=\left(\beta_{01}(t), \ldots, \beta_{0 p}(t)\right)^{\top}$ is a $p$-dimensional smooth function of $t$.

Without loss of generality, we assume $\boldsymbol{\beta}_{0}(t)=\left(\boldsymbol{\beta}_{O}^{\top}(t), \boldsymbol{\beta}_{C}^{\top}(t), \boldsymbol{\beta}_{N C}^{\top}(t)\right)^{\top}$, where $\boldsymbol{\beta}_{O}(t) \in \operatorname{IR}^{p 1}$, $\boldsymbol{\beta}_{C}(t) \in \mathrm{IR}^{p 2}$ and $\boldsymbol{\beta}_{N C}(t) \in \mathrm{IR}^{p 3}$ correspond to covariates with null effect, constant effect and truly time-varying effect, respectively, and $p=p_{1}+p_{2}+p_{3}$. Denote the corresponding index sets of the above three classes by $\mathrm{I}_{O}, \mathrm{I}_{C}$ and $\mathrm{I}_{N C}$, and let $\mathrm{I}=\{1, \ldots, p\}=\left\{\mathrm{I}_{O} \mathrm{UI}_{C} \mathrm{UI}_{N C}\right\}$. Our method consists of two steps. In Step 1 , for any fixed $t$, we obtain the initial estimator $\tilde{\boldsymbol{\beta}}(t)=$ $\left(\tilde{\beta}_{1}(t), \ldots, \tilde{\beta}_{p}(t)\right)^{\top} \in \operatorname{IR}^{p}$ using the kernel-weighted partial likelihood estimation (Cai and Sun, 2003; Tian et al., 2005), i.e., by maximizing the local partial likelihood 


$$
L_{1 n}(\boldsymbol{\beta}, t)=\frac{1}{n} \sum_{i=1}^{n} \int_{0}^{\tau} K_{h}(s-t)\left[\boldsymbol{\beta}^{\top} \boldsymbol{Z}_{i}-\log \left(\sum_{j=1}^{n} Y_{j}(s) e^{\boldsymbol{\beta}^{\top} \boldsymbol{Z}_{j}}\right)\right] d N_{i}(s)
$$

with respect to $\beta$, where $K_{h}(\cdot)=K(\cdot / h) / h$ with $K(\cdot)$ being a symmetric kernel density function and $h$ being the bandwidth parameter, $Y_{j}(t)=\mathbb{1}\left(\tilde{T}_{j} \geq t\right), N_{i}(t)=\mathbb{1}\left(\tilde{T}_{i} \leq t, \Delta_{i}=1\right)$, and $\tau$ is a pre-specified constant such that $P\left(\tilde{T}_{i}>\tau\right)>0$. Next, we decompose the initial estimator $\tilde{\boldsymbol{\beta}}(t)$ into the mean part $\tilde{\boldsymbol{m}}=\left(\tilde{m_{1}}, \ldots, \tilde{m_{p}}\right)^{\top}$ and the deviation part $\tilde{\boldsymbol{\beta}}^{*}(t)=\left(\tilde{\beta}_{1}^{*}(t), \ldots, \tilde{\beta}_{p}^{*}(t)\right)^{\top}$, where $\tilde{m}_{k}=\tau^{-1} \int_{0}^{\tau} \tilde{\beta}_{k}(u) d u$ and $\tilde{\beta}_{k}^{*}(t)=\tilde{\beta}_{k}(t)-\tilde{m}_{k}$ for $k=1, \ldots$, $p$. Practically, we could choose $M$ grid points $\mathscr{T}_{M}=\left\{t_{1}, \ldots, t_{M}\right\}$, equally spaced between 0 and $\tau$, where $M$ is a large positive integer. We then let $\tilde{m}_{k}=\sum_{i=1}^{M} \tilde{\beta}_{k}\left(t_{i}\right) / M$ and $\tilde{\beta}_{k}^{*}(t)=\tilde{\beta}_{k}\left(t_{j}\right)-\tilde{m}_{k}$, where $j=\operatorname{argmin}_{k} / t_{k}-t /$ and $t \in[0, \tau]$. In our numerical studies, we set $M$ to be 100 , which based on our experiment is large enough to make good approximation of both $\tilde{n}_{k}$ and $\tilde{\beta}_{k}^{*}(t)$.

In Step 2, we adapt group nonnegative garrote penalties for structure selection. Denote $\lambda_{1}=$ $\left(\lambda_{11}, \ldots, \lambda_{1 p}\right)^{\top}, \lambda_{2}=\left(\lambda_{21}, \ldots, \lambda_{2 p}\right)^{\top}$ as two $p$-dimensional vectors. We obtain $\hat{\lambda_{1}}$ and $\hat{\lambda_{2}}$ by minimizing

$$
Q_{2 n}\left(\boldsymbol{\lambda}_{1}, \boldsymbol{\lambda}_{2}\right)=-\sum_{i=1}^{n} \int_{0}^{\tau}\left[\left(\tilde{\boldsymbol{m}} \circ \boldsymbol{\lambda}_{1}+\tilde{\boldsymbol{\beta}}^{*}(s) \circ \boldsymbol{\lambda}_{2}\right)^{\top} \boldsymbol{Z}_{i}-\log \left(\sum_{j=1}^{n} Y_{j}(s) e^{\left(\tilde{\boldsymbol{m}} \circ \boldsymbol{\lambda}_{1}+\tilde{\boldsymbol{\beta}}^{*}(s) \circ \boldsymbol{\lambda}_{2}\right)^{\top} \boldsymbol{Z}_{j}}\right) d N_{i}(s)+\theta_{1} \sum_{j=1}^{p} \lambda_{1 j}+\theta_{2} \sum_{j=1}^{p} \lambda_{2 j}\right.
$$

subject to $\lambda_{1 j} \geq 0$ and $\lambda_{2 j} \geq 0, j=1, \cdots, p$, where $\theta=\left(\theta_{1}, \theta_{2}\right)$ are two-dimensional nonnegative tuning parameters, and $a \circ b$ denotes the Hadamard (element-wise) product of vectors $a$ and $b$. Then, the proposed KGNG estimator of $\beta_{0 k}(t)$ is given by

$$
\hat{\beta}_{k}(t)=\hat{\lambda}_{1 k} \tilde{m}_{k}+\hat{\lambda}_{2 k} \tilde{\beta}_{k}^{*}(t), k=1, \ldots, p, t \in[0, \tau] .
$$

It is interesting to note that the automatic structure selection is achieved by shrinking some components of $\hat{\lambda_{1}}$ and $\hat{\lambda_{2}}$ to zero. Specifically, we define $\hat{\mathrm{I}}_{O}=\left\{k \in \mathrm{I}: \hat{\lambda_{1 k}}=0, \hat{\lambda_{2 k}}=0\right\}, \hat{\mathrm{I}}_{C}$ $=\left\{k \in \mathrm{I}: \hat{\lambda_{1 k}} \neq 0, \hat{\lambda_{2 k}}=0\right\}$ and $\hat{\mathrm{I}}_{N C}=\left\{k \in \mathrm{I}: \hat{\lambda_{2 k}} \neq 0\right\}$ as estimated index sets for $\mathrm{I}_{O}, \mathrm{I}_{C}$ and $\mathrm{I}_{N C}$, respectively.

When the number of covariates is large, the inclusion of many noise variables (covariates with null effect) at Step 1 may affect the structure selection performance. Next, we propose a variant of KGNG estimator. Specifically, we add a preliminary step (Step 0) before Steps 1 
and 2 to exclude all noise variables prior to structure selection. To do this, we conduct a standard group nonnegative garrote estimation by minimizing

$$
-\sum_{i=1}^{n} \int_{0}^{\tau}\left[\left(\tilde{\boldsymbol{\beta}}(s) \circ \boldsymbol{\lambda}^{*}\right)^{\top} \boldsymbol{Z}_{i}-\log \left(\sum_{j=1}^{n} Y_{j}(s) e^{\left(\tilde{\boldsymbol{\beta}}(s) \circ \boldsymbol{\lambda}^{*}\right)^{\top} \boldsymbol{Z}_{j}}\right)\right] d N_{i}(s)+\theta^{*} \sum_{j=1}^{p} \lambda_{j}^{*}
$$

with respect to $\lambda^{*}=\left(\lambda_{1}^{*}, \ldots, \lambda_{p}^{*}\right)^{\top}$, where $\lambda_{j}^{*} \geq 0, j=1, \ldots, p$, and $\theta^{*}$ a nonnegative tuning parameter. Let $\hat{\lambda}^{*}=\left(\hat{\lambda}_{1}^{*}, \ldots, \hat{\lambda}_{p}^{*}\right)^{\top}$ denote the resulting minimizer. We exclude the $k$ th covariate if $\hat{\lambda}_{k}^{*}=0$. Let $\underline{\boldsymbol{Z}}_{i}$ denote the remaining sub-vector of $\boldsymbol{Z}_{i}$ by keeping all important covariates. We then implement Steps 1 and 2 with $\boldsymbol{Z}_{i}$ replaced by $\underline{\boldsymbol{Z}}_{i}$ for further structure selection. The resulting estimator is denoted as KGNG2.

\subsection{Computational Aspects}

We implemented the proposed method in $\mathrm{R}$, and the corresponding code can be downloaded from the author's web page (http://www4.ncsu.edu/ wxiao/). In Step $1, L_{1 n}(\boldsymbol{\beta}, t)$ is strictly concave with probability one and thus has a unique solution. The maximization can be realized based on a regular Newton-Raphson iteration or an efficient iterative algorithm proposed in Cai et al. (2000). In Step 2, after proper transformations, the minimization problem of (2.3) is equivalent to finding the lasso solution for a Cox model with timedependent covariates $\tilde{Z_{I}}(s)$ under the nonnegative constraint of regression parameters, where

$$
\tilde{\boldsymbol{Z}}_{i}(s)=\left(\begin{array}{c}
\tilde{\boldsymbol{m}} \circ \boldsymbol{Z}_{i} \\
\tilde{\boldsymbol{\beta}}^{*}(s) \circ \boldsymbol{Z}_{i}
\end{array}\right)
$$

We used R package "penalized" (Goeman, 2010) for this optimization step. The algorithm is based on a combination of gradient ascent optimization and Newton-Raphson algorithm, which can also incorporate nonnegative constraints on the parameters (Goeman, 2010). Moreover, the minimization in the preliminary Step 0 is also equivalent to finding a lasso solution for a Cox model with time-dependent covariates $\tilde{\boldsymbol{\beta}}(s) \circ \boldsymbol{Z}_{i}$. Therefore it can be computed similarly with existing $\mathrm{R}$ packages.

\subsection{Tuning Procedure}

For computing KGNG, two sets of tuning parameters need to be chosen properly, i.e., the bandwidth $h$ at the maximum local partial likelihood estimation step (2.2) and $\left(\theta_{1}, \theta_{2}\right)$ at the group nonnegative garrote estimation step (2.3). To choose $h$, we use a $K$-fold crossvalidation method as suggested in Tian et al. (2005). First, we randomly split the data set into $K$ roughly equal-sized parts. Then, for each $k=1, \ldots, K$, we delete the $k$ th part and fit the time-varying coefficient Cox model with the other $K-1$ parts. Next, we compute the prediction error $P E_{k}(h)$, which measures how well the fitted model predicts the $k$ th part of the data. Here, 


$$
P E_{k}(h)-\sum_{i \in \mathrm{I}_{k}} \int_{0}^{\tau}\left[\tilde{\boldsymbol{\beta}}^{(-k)}(s)^{\top} \boldsymbol{Z}_{i}-\log \left(\sum_{j \in \mathrm{I}_{k}} Y_{j}(s) e^{\tilde{\boldsymbol{\beta}}^{(-k)}(s)^{\top} \boldsymbol{Z}_{j}}\right)\right] d N_{i}(s),
$$

where $\mathrm{I}_{k}$ is the index set for the $k$ th part of the data and $\tilde{\boldsymbol{\beta}}^{(-k)}(t)$ is the maximum local partial likelihood estimator calculated with the $k$ th part of the data deleted. Last, the optimal $h$ is obtained by minimizing the total prediction error $P E(h)=\sum_{k=1}^{K} P E_{k}(h)$.

For $\left(\theta_{1}, \theta_{2}\right)$, we consider a set of bivariate grid values, and choose the optimal $\left(\theta_{1}, \theta_{2}\right)$ by minimizing the following BIC criterion

$$
\mathbf{B I C}=-2 \log (\text { partial likelihood }) / n+\log n / n \times d f_{1}+\log \left(n h^{*}\right) /\left(n h^{*}\right) \times d f_{2},
$$

where $d f_{1}$ and $d f_{2}$ are the number of nonzero components in $\hat{\lambda_{1}}$ and $\hat{\lambda_{2}}$, respectively, and $h^{*}$ $=h / \tau$ is the effective bandwidth when we scale $\tau$ to 1 . Note that the effective sample size $n h^{*}$ is used here for the time-varying components instead of the original $n$ to account for the fact that $\beta_{i}^{*}(t)$ is estimated locally. Similar strategy is adopted in Wang and Xia (2009) and $\mathrm{Hu}$ and Xia (2012).

Similarly, $\theta^{*}$ in the preliminary Step 0 can be chosen by minimizing the BIC criterion

$$
\mathbf{B I C}=-2 \log (\text { partial likelihood }) / n+\log \left(n h^{*}\right) /\left(n h^{*}\right) \times d f_{3},
$$

where $d f_{3}$ is the number of covariates with nonzero effect.

\section{Theoretical properties}

In this section, we establish the asymptotic properties of the initial estimators, and our proposed KGNG and KGNG2 estimators.

\subsection{Asymptotic Properties of Initial Estimators}

Denote the true mean and deviation part of $\boldsymbol{\beta}_{0}(t)$ as $\boldsymbol{m}_{0}=\left(m_{01}, \ldots, m_{0 p}\right)^{\top}$ and $\boldsymbol{\beta}_{0}^{*}(t)=\left(\beta_{01}^{*}(t), \ldots, \beta_{0 p}^{*}(t)\right)^{\top}$, respectively, where

$$
m_{0 k}=\tau^{-1} \int_{0}^{\tau} \beta_{0 k}(u) d u, \quad \beta_{0 k}^{*}(t)=\beta_{0 k}(t)-m_{0 k},
$$

for $k=1, \ldots, p$. Let $I(\boldsymbol{\beta}, t)=-\partial^{2} L_{1 n}(\boldsymbol{\beta}, t) / \partial \boldsymbol{\beta}^{2}=n^{-1} \sum_{i=1}^{n} \int_{0}^{\tau} V(\boldsymbol{\beta}, s) K_{h_{n}}(s-t) d N_{i}(s)$, where 


$$
\begin{array}{cc}
V(\boldsymbol{\beta}, t) & =\frac{S^{(2)}(\boldsymbol{\beta}, t)}{S^{(0)}(\boldsymbol{\beta}, t)}-\left(\frac{S^{(1)}(\boldsymbol{\beta}, t)}{S^{(0)}(\boldsymbol{\beta}, t)}\right)^{\otimes 2}, \\
S^{(r)}(\boldsymbol{\beta}, t) & =n^{-1} \sum_{i=1}^{n} Y_{i}(t) \boldsymbol{Z}_{i}^{\otimes r} e^{\boldsymbol{\beta}^{\prime} \boldsymbol{Z}_{i}}, r=0,1,2,
\end{array}
$$

with $\otimes$ denoting the outer product. Let $\boldsymbol{E}(\boldsymbol{\beta}, t)=S^{(1)}(\boldsymbol{\beta}, t) / S^{(0)}(\boldsymbol{\beta}, t), P(t / z)=\mathrm{P}(\tilde{T} \geq t \boldsymbol{Z}=\boldsymbol{z})$, $Q_{0}(t)=\mathrm{E}[P(t / Z) a(t / Z)], Q_{1}(t)=\mathrm{E}[P(t / \mathbb{Z}) a(t / \mathbb{Z}) Z]$ and $Q_{2}(t)=\mathrm{E}\left[P(t / Z) a(t / Z) Z^{\otimes 2}\right]$. Define $\Sigma(t)=Q_{2}(t)-Q_{1}(t) Q_{1}(t)^{\top} / Q_{0}(t)$. Let $s^{(r)}(\boldsymbol{\beta}, t)$ denote the limits of $S^{(r)}(\boldsymbol{\beta}, t), r=0,1,2$, as $n$ $\rightarrow \infty$. Let $\mathscr{N}(t, \mathcal{\varepsilon})$ be an $\mathcal{\varepsilon}$-neighborhood of $t$, for $\mathcal{\varepsilon}>0$ and $t \in[0, \tau]$, and $\mathbb{B}$ be a compact set of $\operatorname{IR}^{p}$ that includes a neighborhood of $\beta_{0}(t)$ for $t \in[0, \tau]$. Under the following regularity conditions,

(A.1) The kernel function $K(\cdot)$ is a bounded and symmetric density with a bounded support $[-1,1]$;

(A.2) For $t \in[0, \tau]$

$$
\mathrm{E}\left[\exp \left\{2\left(\sup _{u \in \mathscr{N}(t, \varepsilon)}\left|\boldsymbol{\beta}_{0}(u)\right|+\boldsymbol{\beta}_{0}^{\prime}(t)+3\right)|Z|\right\}\right]<\infty
$$

(A.3) $Q_{0}(t)>0, Q_{1}(t)$ and $Q_{2}(t)$ are continuous for $t \in[0, \tau]$;

(A.4) Assume that $a_{0}(t)$ is positive and continuous, $P(t /)>0$ and coefficient functions $\left\{\beta_{0 j}(t)\right\}$ have a continuous second derivative for $t \in[0, \tau]$;

(A.5) Assume that the matrix $\Sigma(t)$ is positive definite for $t \in[0, \tau]$;

(A.6) $s^{(r)}(\boldsymbol{\beta}, t)$ is uniformly continuous with respect to $\left(\boldsymbol{\beta}^{\top}, t\right)^{\top} \in \mathbb{R} \times[0, \tau]$ for $t \in$ $[0, \tau]$

we have

Lemma 1-Suppose $h_{n}=O\left(n^{-v}\right)$ with $v \in[1 / 5,1)$.

a.

b. $\quad$ If $1 / 5<v<1$, we have, for fixed $t \in(0, \tau)$,

$$
\left(n h_{n}\right)^{1 / 2}\left(\tilde{\boldsymbol{\beta}}(t)-\boldsymbol{\beta}_{0}(t)\right) \stackrel{d}{\rightarrow} N\left\{0, \sum^{-1}(t) \int_{-1}^{1} K^{2}(s) d s\right\}
$$

where $\Sigma(t)$ can be consistently estimated by $I(\tilde{\beta}(t), t)$.

Lemma 2-Suppose $h_{n}=O\left(n^{-v}\right)$ with $1 / 4<v<1 / 2$, we have

$$
n^{1 / 2}\left(\tilde{\boldsymbol{m}}-\boldsymbol{m}_{0}\right) \stackrel{d}{\rightarrow} N\left(0, \sum_{m}\right),
$$


where $\sum_{m}=\int_{0}^{\tau} \sum^{-1}(u) d u / \tau^{2}$ and can be consistently estimated by

$$
\hat{\sum}_{m}=\int_{h}^{\tau-h} I^{-1}(\tilde{\beta}(u), u) d u /(\tau-2 h)^{2} .
$$

The proof of Lemma 1 follows Cai and Sun (2003) and the proof of Lemma 2 follows similar steps given in Section 5 of Tian et al. (2005), which are omitted.

\subsection{Asymptotic Properties of KGNG Estimators}

Let $\lambda_{01}$ and $\boldsymbol{\lambda}_{02}$ denote the true values of $\boldsymbol{\lambda}_{1}$ and $\boldsymbol{\lambda}_{2}$, respectively, and partition them into three parts: $\boldsymbol{\lambda}_{01}=\left(\boldsymbol{\lambda}_{01}^{O^{\top}}, \boldsymbol{\lambda}_{01}^{C^{\top}}, \boldsymbol{\lambda}_{01}^{N C^{\top}}\right)^{\top}$ and $\boldsymbol{\lambda}_{02}=\left(\boldsymbol{\lambda}_{02}^{O^{\top}}, \boldsymbol{\lambda}_{02}^{C^{\top}}, \boldsymbol{\lambda}_{02}^{N C^{\top}}\right)^{\top}$, according to the true index sets $\mathrm{I}_{O}, \mathrm{I}_{C}$ and $\mathrm{I}_{N C}$, respectively. Then

$$
\lambda_{01}^{O}=\mathbf{0}_{p_{1}}, \quad \lambda_{01}^{C}=\mathbf{1}_{p_{2}}, \quad \lambda_{02}^{O}=\mathbf{0}_{p_{1}}, \quad \lambda_{02}^{C}=\mathbf{0}_{p_{2}}, \quad \lambda_{02}^{N C}=\mathbf{1}_{p_{3}}
$$

and $\lambda_{01, j}^{N C}=0$ or 1 for $j \in \mathrm{I}_{N C}$, where $\lambda_{01, j}^{N C}$ is the $j$ th component of $\boldsymbol{\lambda}_{01}^{N C}$ and corresponds to the mean part of the $j$ h covariate with time-varying effect. If $\lambda_{01, j}^{N C}=0$, the $j$ th time-varying effect has zero mean effect; otherwise, it has nonzero mean effect. As we do not distinguish between the above two types of time-varying effects, without loss of generality, we assume $\boldsymbol{\lambda}_{01}^{N C}=\mathbf{1}_{p_{3}}$ in our theoretical derivations. Let $\boldsymbol{\lambda}_{0}=\left(\boldsymbol{\lambda}_{01}^{\top}, \boldsymbol{\lambda}_{02}^{\top}\right)^{\top}$. We further partition $\boldsymbol{\lambda}_{0}$ as $\boldsymbol{\lambda}_{0}^{(1)}$ representing all the ones and $\boldsymbol{\lambda}_{0}^{(0)}$ representing all the zeros, where

$\boldsymbol{\lambda}_{0}^{(1)}=\left(\boldsymbol{\lambda}_{01}^{C^{\top}}, \boldsymbol{\lambda}_{01}^{N C^{\top}}, \boldsymbol{\lambda}_{02}^{N C^{\top}}\right)^{\top}=\mathbf{1}_{p_{2}+2 p_{3}}$ and $\boldsymbol{\lambda}_{0}^{(0)}=\left(\boldsymbol{\lambda}_{01}^{O^{\top}}, \boldsymbol{\lambda}_{02}^{O^{\top}}, \boldsymbol{\lambda}_{02}^{C^{\top}}\right)^{\top}=\mathbf{0}_{2 p_{1}+p_{2}}$. In a similar manner we define $\boldsymbol{\lambda}, \boldsymbol{\lambda}^{(1)}, \boldsymbol{\lambda}^{(0)}, \hat{\boldsymbol{\lambda}}, \hat{\boldsymbol{\lambda}}^{(1)}$ and $\hat{\boldsymbol{\lambda}}^{(0)}$.

Note that the KGNG estimator defined in (2.4) takes the form $\hat{\boldsymbol{\beta}}(t)=\hat{\boldsymbol{\lambda}_{1}}{ }^{\circ} \tilde{\boldsymbol{m}}+\hat{\boldsymbol{\lambda}_{2}} \circ \widetilde{\boldsymbol{\beta}}^{*}(t)$. To derive its asymptotic properties, we need to study the asymptotic properties of $\tilde{\boldsymbol{m}}, \widetilde{\boldsymbol{\beta}}(t)$ and $\hat{\boldsymbol{\lambda}}$. In Lemmas 1 and 2, we have established the asymptotic properties of $\tilde{\boldsymbol{m}}$ and $\widetilde{\boldsymbol{\beta}}(t)$. In the following, we derive the asymptotic properties of $\hat{\lambda}$, i.e., $\hat{\lambda}^{(1)}$ and $\hat{\lambda}^{(0)}$. Specifically, we establish the root- $n$ consistency of $\hat{\lambda}^{(1)}$ in Theorem 1 and the sparsity property of $\hat{\lambda}^{(0)}$ in Theorem 2.

Theorem 1-Assume $h_{n}=O\left(n^{-v}\right)$ with $1 / 4<v<1 / 2$. Under the regularity conditions assumed in Lemmas 1 and 2, if $\max \left(\theta_{1}, \theta_{2}\right) / \sqrt{n}$ is bounded, then $\left\|\hat{\boldsymbol{\lambda}}^{(1)}-\boldsymbol{\lambda}_{0}^{(1)}\right\|=O_{p}\left(n^{-1 / 2}\right)$.

Theorem 2-Assume $h_{n}=O\left(n^{-v}\right)$ with $1 / 4<v<1 / 2$. Under the regularity conditions assumed in Lemmas 1 and 2 , if $\left\|\hat{\boldsymbol{\lambda}}^{(1)}-\boldsymbol{\lambda}_{0}^{(1)}\right\|=O_{p}\left(n^{-1 / 2}\right)$ and $h_{n}^{1 / 2} \min \left(\theta_{1}, \theta_{2}\right) \rightarrow \infty$, then $P$ $\left(\hat{\boldsymbol{\lambda}}^{(0)}=\mathbf{0}\right) \rightarrow 1$.

Combining Theorems 1 and 2, we can then prove the selection consistency of the KGNG estimator, which is summarized in part (a) of Theorem 3. We further establish the 
asymptotic normality of the KGNG estimators for both nonzero constant and time-varying regression coefficients in Theorem 3. Recall that $\boldsymbol{\beta}_{0}(t)=\left(\boldsymbol{\beta}_{O}^{\top}, \boldsymbol{\beta}_{C}^{\top}, \boldsymbol{\beta}_{N C}^{\top}(t)\right)^{\top} \in \mathbb{R}^{p}$, where $\boldsymbol{\beta}_{O}$ $\in \operatorname{IR}^{p 1}, \boldsymbol{\beta}_{C} \in \operatorname{IR}^{p 2}$ and $\boldsymbol{\beta}_{N C}(t) \in \operatorname{IR}^{p 3}$ are subvector of $\boldsymbol{\beta}_{0}(t)$ corresponding to the true underline index sets $\mathrm{I}_{O}, \mathrm{I}_{C}$ and $\mathrm{I}_{N C}$, respectively. In a similar manner, we write

$\boldsymbol{m}_{0}=\left(\boldsymbol{m}_{O}^{\top}, \boldsymbol{m}_{C}^{\top}, \boldsymbol{m}_{N C}^{\top}\right)^{\top}$ and $\boldsymbol{\beta}_{0}^{*}(t)=\left(\beta_{O}^{* \top}, \boldsymbol{\beta}_{C}^{* \top}, \boldsymbol{\beta}_{N C}^{*}{ }^{\top}(t)\right)^{\top}$. We further partition $\hat{\boldsymbol{\beta}}(t), \tilde{\boldsymbol{\beta}}(t)$, $\tilde{\boldsymbol{m}}$ and $\tilde{\boldsymbol{\beta}}^{*}(t)$ accordingly.

Theorem 3-Assume $h_{n}=O\left(n^{-v}\right)$ with $1 / 4<v<1 / 2$. Under the regularity conditions assumed in Lemmas 1 and 2, if $\max \left(\theta_{1}, \theta_{2}\right) / \sqrt{n}$ is bounded and $h_{n}^{1 / 2} \min \left(\theta_{1}, \theta_{2}\right) \rightarrow \infty$, then

a. (Selection consistency) with probability tending to one, $\hat{\mathrm{I}}_{O}=\mathrm{I}_{O}, \hat{\mathrm{I}}_{C}=\mathrm{I}_{C}$ and $\hat{\mathrm{I}}_{N C}=\mathrm{I}_{N C}$.

b. (Root-n consistency of $\left.\hat{\boldsymbol{\beta}}_{C}\right) \hat{\boldsymbol{\beta}}_{C}$ is a root-n consistent estimator for $\boldsymbol{\beta}_{C}$.

c. (Asymptotic normality of $\hat{\boldsymbol{\beta}}_{C}$ if we further assume $\max \left(\theta_{1}, \theta_{2}\right) / \sqrt{n} \rightarrow 0$,

$$
(n)^{1 / 2}\left(\hat{\boldsymbol{\beta}}_{C}-\boldsymbol{\beta}_{C}\right) \stackrel{d}{\rightarrow} N\left\{0, \sum_{m}^{F}\right\}
$$

where

$$
\sum_{m}^{F}=\int_{0}^{\tau}\left(D(u)+\frac{1}{\tau} B_{10} \sum^{-1}(u)\right) \sum(u)\left(D(u)+\frac{1}{\tau} B_{10} \sum^{-1}(u)\right)^{\top} d u .
$$

Here $D(u)$ is a $p_{2} \times p$ matrix given in $(\mathrm{S} 3.8)$ in the Supplement and $B_{10}=$ $\left(\mathbf{0}_{p_{2} \times p_{1}}\left|\boldsymbol{I}_{p_{2}}\right| \mathbf{0}_{p_{2} \times p_{3}}\right)$.

d.

(Asymptotic normality of $\hat{\beta}_{N C}(t)$ )

$$
\left(n h_{n}\right)^{1 / 2}\left(\hat{\boldsymbol{\beta}}_{N C}(t)-\boldsymbol{\beta}_{N C}(t)\right) \stackrel{d}{\rightarrow} N\left\{0,\left\{\sum^{-1}(t)\right\}_{N C, N C} \int_{-1}^{1} K^{2}(s) d s\right\},
$$

where $\left\{\Sigma^{-1}(t)\right\}_{N C, N C}$ is the submatrix of $\Sigma^{-1}(t)$ corresponding to $\mathrm{I}_{N C}$.

The proofs of Theorem 1, 2 and 3 are relegated to the online supplementary appendix. The asymptotic variance-covariance matrices given in parts (c) and (d) can be consistently estimated by the usual plug-in method. It is interesting to note that the limiting distribution of the KGNG estimator for the time-varying coefficients given in part (d) is the same as that of the corresponding initial estimator. Actually in the proof of part (d), we have shown that the difference between $\hat{\boldsymbol{\beta}}_{N C}(t)$ and $\tilde{\boldsymbol{\beta}}_{N C}(t)$ are uniformly asymptotically negligible in $t$. Thus 
we could construct confidence bands of $\hat{\boldsymbol{\beta}}_{N C}(t)$ using the resampling technique as proposed in Tian et al. (2005).

\subsection{Asymptotic Properties of KGNG2 Estimator}

Let $\boldsymbol{Z}=\left(\boldsymbol{Z}_{O}^{\top}, \boldsymbol{Z}_{C}^{\top}, \boldsymbol{Z}_{N C}^{\top}\right)^{\top}$ and $\underline{\boldsymbol{Z}}=\left(\boldsymbol{Z}_{C}^{\top}, \boldsymbol{Z}_{N C}^{\top}\right)^{\top}$ as the subvector of $\boldsymbol{Z}$ with only important covariates kept. Let $\underline{\boldsymbol{\beta}}_{0}(t)=\left(\boldsymbol{\beta}_{C}^{\top}(t), \boldsymbol{\beta}_{N C}^{\top}(t)\right)^{\top}, \alpha(t \mid \underline{\boldsymbol{Z}})=\alpha_{0}(t) e^{\underline{\boldsymbol{\beta}}_{0}(t)^{\top} \underline{\boldsymbol{Z}}}, P(t \mid \underline{\boldsymbol{Z}})=P(Y \geq t \mid \underline{\boldsymbol{Z}}=$ $\underline{z}), \underline{Q}_{0}(t)=E[P(t \mid \underline{\boldsymbol{Z}}) a(t \mid \underline{\boldsymbol{Z}})], \underline{Q}_{1}(t)=E[P(t \underline{\boldsymbol{Z}}) a(t \mid \underline{\boldsymbol{Z}}) \underline{\boldsymbol{Z}}]$ and $\underline{Q}_{2}(t)=E\left[P(t \mid \underline{\boldsymbol{Z}}) a(t \mid \underline{\boldsymbol{Z}})^{\otimes 2}\right]$. Define $\underline{\Sigma}(t)=\underline{Q}_{2}(t)-\underline{Q}_{1}(t) \underline{Q}_{1}(t)^{\top} / \underline{Q}_{0}(t)$. We add “*” to distinguish the KGNG2 estimator from the KGNG estimator. We summarize the asymptotic properties of KGNG2 estimator in Theorem 4.

Theorem 4-Assume $h_{n}=O\left(n^{-v}\right)$ with $1 / 4<v<1 / 2$. Under the regularity conditions assumed in Lemmas 1 and 2, if (1) $\theta^{*} / \sqrt{n}$ is bounded and $h_{n}^{1 / 2} \theta^{*} \rightarrow \infty$; (2) $\max \left(\theta_{1}, \theta_{2}\right) / \sqrt{n}$ is bounded and $h_{n}^{1 / 2} \min \left(\theta_{1}, \theta_{2}\right) \rightarrow \infty$, then

a. (Selection consistency of preliminary Step 0) with probability tending to one, $\hat{\lambda}_{k}^{*}=0$, for $k \in \mathrm{I}_{O}$ and $\hat{\lambda}_{k}^{*} \neq 0$, for $k \in \mathrm{I}_{C} \cup \mathrm{I}_{N C}$.

b. (Selection consistency) with probability tending to one, $\hat{\mathrm{I}}_{O}^{*}=\mathrm{I}_{O}, \hat{\mathrm{I}}_{C}^{*}=\mathrm{I}_{C}$ and $\hat{\mathrm{I}}_{N C}^{*}=I_{N C}$.

c. $\left(\boldsymbol{R o o t}-\boldsymbol{n}\right.$ consistency of $\left.\hat{\boldsymbol{\beta}}_{C}^{*}\right) \hat{\boldsymbol{\beta}}_{C}^{*}(t)$ is a root-n consistent estimator for $\beta_{C}$.

d.

(Asymptotic normality of $\left.\hat{\boldsymbol{\beta}}_{C}^{*}\right)$ if we further assume $\max \left(\theta_{1}, \theta_{2}\right) / \sqrt{n} \rightarrow 0$,

$$
(n)^{1 / 2}\left(\hat{\boldsymbol{\beta}}_{C}^{*}-\boldsymbol{\beta}_{C}\right) \stackrel{d}{\rightarrow} N\left\{0, \underline{\Sigma}_{m}^{F}\right\}
$$

where $\sum_{m}^{F}$ can be computed following (3.1) with some obvious changes.

e. (Asymptotic normality of $\hat{\boldsymbol{\beta}}_{N C}^{*}(t)$ )

$$
\left(n h_{n}\right)^{1 / 2}\left(\hat{\boldsymbol{\beta}}_{N C}^{*}(t)-\boldsymbol{\beta}_{N C}(t)\right) \stackrel{d}{\rightarrow} N\left\{0,\left\{\underline{\sum}^{-1}(t)\right\}{ }_{N C, N C} \int_{-1}^{1} K^{2}(s) d s\right\},
$$

where $\left\{\underline{\Sigma}^{-1}(t)\right\}_{N C, N C}$ is the submatrix of $\underline{\Sigma}^{-1}(t)$ corresponding to $\mathrm{I}_{N C}$.

The proof of Theorem 4 is similar to that of Theorem 3 and hence is omitted here. Based on Theorem 3 and $4, \hat{\boldsymbol{\beta}}_{N C}^{*}(t)$ is strictly more efficient than $\hat{\boldsymbol{\beta}}_{N C}(t)$ if $\mathrm{I}_{O}$ is not empty. However, there is no clear order between the efficiencies of $\hat{\boldsymbol{\beta}}_{C}^{*}$ and $\hat{\boldsymbol{\beta}} C$. 


\section{Numerical studies}

\subsection{Simulation studies}

We generate failure times from the varying-coefficient Cox model (2.1). Here, covariate vector $\boldsymbol{Z}$ is generated from a multivariate normal distribution with mean 0 , variance 0.5 and correlation coefficient $0.5^{j-k /}$ for any pair $(j, k)$. We consider both the low dimensional case and the high dimensional case, where the dimension $p=10$ and 50 respectively. There are three nonzero coefficients in $\beta_{0}(t)$, i.e., $\beta_{02}(t)=-\{1+\cos (\pi t)\} \mathbb{1}(0<t<1), \beta_{03}(t)=$ $1.5\{\cos (\pi t / 2)\}$ and $\beta_{08}(t)=-1$. So, there are two covariates with time-varying effects, one with constant effect and all the remains with null effect. The baseline hazard function $a_{0}(t)=$ $\exp \{-\cos (\pi t / 2)\}$. We consider both the cases when censoring times are dependent and independent of the covariates. They are given in the cases $p=10$ and $p=50$, respectively. When $p=10$, the censoring times of $t$ th subject is a mixture of $W$ and a point mass at 2, where $W=\min \left(\exp \left(Z_{i 2}-Z_{i 5}\right), \operatorname{Unif}(0,2)\right)$. When $p=50$, we generate censoring times from a mixture of $\operatorname{Unif}(0,2))$ and a point mass at 2 . For both cases, the mixing probability is chosen to have the desired censoring proportion, i.e. $c_{p}=20 \%$ or $40 \%$. For each scenario, we conduct 100 simulation runs with sample size $n=200$ and 400. We compare the proposed KGNG and KGNG2 with the AGLASSO of Yan and Huang (2012). For our estimators, we use the Epanechnikov kernel $K(x)=3\left(1-x^{2}\right) / 4,-1 \leq x \leq 1$. The bandwidth $h$ is chosen using 5 -fold cross validation as discussed in Section 2.3. For KGNG2, the same bandwidth is used for Step 1 as for Step 0. We use the proposed BIC criterion in Section 2.3 to tune $\left(\theta_{1}, \theta_{2}\right)$ and $\theta^{*}$. In addition, we compare the proposed methods with a conventional method based on the confidence bands, denoted as the CB method. Specifically, in the CB method, we first constructed confidence bands based on the initial estimates of time-varying coefficients as done in Tian et al. (2005). If the zero-line is contained in the estimated confidence band, we classify the corresponding covariate as a null-effect covariate. If a constant-line but not the zero-line is contained in the estimated confidence band, we classify the corresponding covariate as a constant-effect covariate. If the above two conditions are both not satisfied, we then classify the corresponding covariate as a time-varying-effect covariate.

Tables 1 and 2 summarize the mean squared errors and variable selection results for $p=10$ and 50, respectively. The selection frequency of each variable over 100 runs is reported, where the important covariates are $Z_{2}, Z_{3}$ and $Z_{8}$. The mean squared error (MSE) is calculated as

$$
\frac{1}{100} \sum_{i=1}^{100}\left\{\hat{\boldsymbol{\beta}}\left(t_{i}\right)-\boldsymbol{\beta}_{0}\left(t_{i}\right)\right\}^{\top} V\left\{\hat{\boldsymbol{\beta}}\left(t_{i}\right)-\boldsymbol{\beta}_{0}\left(t_{i}\right)\right\},
$$

where $\left\{t_{1}, \ldots, t_{100}\right\}$ are 100 equally-spaced grid points in the time interval $(0,2)$ and $V$ is the population covariance matrix of covariates.

From Tables 1 and 2, we make the following observations. First, KGNG2 shows the best performance in terms of variable selection and MSE in almost all scenarios, especially for 
high dimension case $(p=50)$. This is expected since KGNG2 is a two-stage approach, by first excluding the noise variables. Second, both KGNG and KGNG2 outperform AGLASSO with deduction in MSE as large as $60 \%$. Third, KGNG and KGNG2 select $Z_{2}, Z_{3}$ and $Z_{8}$ as important covariates nearly all the times, while the AGLASSO misses $Z_{3}$ occasionally, especially when the sample size is small. Fourth, when $p$ is moderate $(p=10)$, the CB method overall has comparable performance as the proposed methods except for the case with smaller sample size $(n=200)$ and larger censoring proportion $\left(c_{p}=40 \%\right)$, where the CB method misses the important covariates frequently over simulations; while when $p$ is large $(p=50)$, the performance of the CB method is very bad since it can not identify any important covariates for almost all the simulations.

Table 3 summarizes the structure selection result of the three covariates with nonzero effect. We report the frequencies of each covariate being classified into the three categories $\mathrm{I}_{O}, \mathrm{I}_{C}$ and $\mathrm{I}_{N C}$. In summary, the proposed KGNG and KGNG2 outperform AGLASSO for all three covariates, exhibiting the most significant improvement for $Z_{2}$. The AGLASSO tends to falsely select the time-varying-effect $Z_{2}$ as a constant-effect covariate. For example, when $p$ $=10, n=200$ and $c_{p}=20 \%$, AGLASSO correctly classifies $Z_{2}$ only 34 times out of 100 , while both KGNG and KGNG2 classify $Z_{2}$ correctly for more than 80 times. Similarly as before, the CB method has very poor structure selection results when $p$ is large $(p=50)$.

Finally, we plot the initial estimator, KGNG and AGLASSO for three nonzero coefficients and their pointwise $95 \%$ confidence intervals based on 100 simulations for $p=10$. The plots for $c_{p}=20 \%$ and $c_{p}=40 \%$ are given in Figures 1 and 2, respectively. We make the following observations. First, the performance of both KGNG and AGLASSO improves substantially when sample size increases. Second, KGNG has smaller biases in the estimation of time-varying coefficients compared with AGLASSO in all the cases, while KGNG and AGLASSO give comparable estimates for the constant coefficient. Third, the improvement of KGNG over the initial estimator for time-varying coefficients is not obvious, however, the improvement for the constant coefficient is significant. This matches with our Theorem 3 parts (c) and (d).

\subsection{Analysis of primary biliary cirrhosis (PBC) data}

As an illustration, we apply our KGNG and KGNG2 methods to analyze the PBC data (Fleming and Harrington, 1991). The data is from the Mayo Clinic trial in primary biliary cirrhosis (PBC) of the liver conducted between 1974 and 1984. The primary biliary cirrhosis is a chronic disease in which the bile ducts in one's liver are slowly destroyed. In the study, 312 out of 424 patients who participate in the randomized trial are eligible for the analysis. There are 17 covariates: $\operatorname{trtmt}=$ treatment (Yes/No), age (in 10 years), gender=female/male, ascites=presence of ascites (Yes/No), hypato=presence of hepatomegaly (Yes/No), spiders $=$ presence of spiders, edema=severity of oedema $(0$ denotes no oedema, 0.5 denotes untreated or successfully treated oedema and 1 denotes unsuccessfully treated oedema), logbili=logarithm of serum bilirubin $(\mathrm{mg} / \mathrm{dl})$, chol=serum cholesterol $(\mathrm{mg} / \mathrm{dl})$, logalb=logarithm of albumin $(\mathrm{gm} / \mathrm{dl})$, copper=urine copper $(\mathrm{mg} / \mathrm{day})$, alk=alkaline phosphatase $(\mathrm{U} / \mathrm{liter})$, sgot=liver enzyme $(\mathrm{U} / \mathrm{ml})$, trig=triglicerides $(\mathrm{mg} / \mathrm{dl})$, platelet=platelets 
per $10^{-3} \mathrm{ml}^{3}$, logprotime=logarithm of prothrombin time (seconds), stage=histologic stage of disease (category: 1, 2, 3 or 4 ).

The model selection of this data set has been previously studied in the context of both Cox model with time-independent coefficients (Tibshirani, 1997; Zhang and Lu, 2007) and Cox model with time-varying coefficients (Tian et al., 2005; Yan and Huang, 2012). To ease the comparison, we analyzed the data of 276 patients with no missingness in covariates and took log transformation of serum bilirubin, albumin and prothrombin time as in Yan and Huang (2012). For our methods, we used the 10 -fold cross validation to find the optimal bandwidth in the initial estimator, which is 2000 (days). We chose $\tau=3200$, which covers around $90 \%$ of observed survival times. Table 4 gives the estimates of coefficients by five methods: the maximum partial likelihood estimator (MPLE), the adaptive LASSO (ALASSO) estimator of Zhang and $\mathrm{Lu}$ (2007) based on a standard Cox model, the AGLASSO, and KGNG and KGNG2 based on a time-varying co-efficient Cox model. The numbers given in parenthesis are the estimated standard errors for important constant coefficients selected by each method. The results for ALASSO and AGLASSO are copied directly from Yan and Huang (2012). We make the following observations. First, ALASSO, AGLASSO, KGNG and KGNG2 all select the same 7 important covariates: age, cooper, edema, logbili, logalb, logprotime and stage. Second, KGNG identifies three covariates with time-varying coefficients and KGNG2 identifies two, in which edema and logprotime are the common covariates identified by both KGNG and KGNG2. On the other hand, AGLASSO only selects logbilli as the covariate with time-varying coefficient. These results partly agree with the findings of Tian et al. (2005), where only 5 covariates: age, edema, logbili, logalb and logprotime, are considered in their time-varying coefficient Cox model, and three covariates: edema, logprotime and logbili, are identified as having time-varying coefficients. Finally, in Figures $3-4$, we plotted the estimated coefficients by the initial step (maximum local partial likelihood estimator), KGNG and KGNG2 for the 7 important covariates and their associated $95 \%$ pointwise confidence intervals and simultaneous confidence bands.

\section{Discussion}

We propose a kernel group nonnegative garrote (KGNG) estimation method and its variant (KGNG2) for automatic structure selection and coefficient estimation in a time-varying coefficient Cox model. We establish the asymptotic properties, including structure selection consistency and asymptotic distributions, of our estimators for both constant and timevarying coefficients. Numerical studies have shown the competitive performance of the proposed methods compared with existing approaches.

In this paper, we only focus on the case with fixed dimension $p$, and $p$ is smaller than the sample size $n$. For $p>n$ case, a penalty term needs to be added to (2.2) to get reasonable initial estimates of the coefficient functions. Then Step 2 and 3 can follow similarly as proposed in this paper. For an ultra-high dimension case, say, $p \gg n$, a screening procedure can be utilized to remove the noisy covariates beforehand. Then the dimension of the model can be decreased to a value that can be handled directly. However, a screening procedure for time-varying coefficient Cox model has not yet been developed in the literature, and it needs further investigation. 
Since the proposed procedure depends on a large number of tuning parameters, it may be useful to develop a statistical test to check the goodness-of-fit of the final estimated model. We think that a cumulative sums of martingale residuals-based goodness-of-fit test can be derived for the final estimated model, following the techniques of Lin et al. (1993). A similar goodness-of-fit test procedure was developed for the Dantzig selector in Cox's proportional hazards model (Antoniadis et al., 2010). This is an interesting topic that needs further investigation.

Another interesting problem, as suggested by a referee, is to extend the proposed methods to domain selection, i.e., to develop a procedure which estimates the coefficients as exactly zero on parts of the time domain, and as nonzero (and time-varying) on the remaining parts. One simple solution would be to chop the coefficient functions evenly into small pieces on the study time domain and then apply the group nonnegative garrote penalty to identify the significant pieces. However, when there are a large number of covariates, this approach may be computationally challenging. The proposed KGNG/KGNG2 methods for structure selection can be regarded as a preliminary step for domain selection since they can help to remove all the covariates with null or constant effects and thus achieve effective dimension reduction. Then, the domain selection can focus only on the selected covariates with truly time-varying coefficients. This is an interesting extension that warrants our future research.

\section{Supplementary Material}

Refer to Web version on PubMed Central for supplementary material.

\section{Acknowledgments}

We thank the Editor, an associate editor, and two reviewers for their constructive comments to improve the paper. We acknowledge support from the National Institutes of Health grant RO1 CA140632 and National Science Foundation grants DMS-1418172, DMS-1309507, and DBI-1261830.

\section{References}

Ahmad I, Leelahanon S, Li Q. Efficient estimation of a semi-parametric partially linear varying coefficient model. Ann Statist. 2005; 33(1):258-283.

Akaike, H. Information theory and an extension of the maximum likelihood principle. Second International Symposium on Information Theory; Tsahkadsor. 1971; Budapest: Akadémiai Kiadó; 1973. p. 267-281.

Andersen PK, Gill RD. Cox's regression model for counting processes: a large sample study. Ann Statist. 1982; 10(4):1100-1120.

Antoniadis A, Fryzlewicz P, Letué F. The Dantzig selector in Cox's proportional hazards model. Scand J Stat. 2010; 37(4):531-552.

Breiman L. Better subset regression using the nonnegative garrote. Technometrics. 1995; 37(4):373384.

Cai Z, Fan J, Li R. Efficient estimation and inferences for varying-coefficient models. J Amer Statist Assoc. 2000; 95(451):888-902.

Cai Z, Sun Y. Local linear estimation for time-dependent coefficients in Cox's regression models. Scand J Statist. 2003; 30(1):93-111.

Cox DR. Regression models and life-tables (with discussions). J Roy Statist Soc Ser B. 1972; 34:187220. 
Fan J, Huang T. Profile likelihood inferences on semiparametric varying-coefficient partially linear models. Bernoulli. 2005; 11(6):1031-1057.

Fan J, Li R. Variable selection via nonconcave penalized likelihood and its oracle properties. J Amer Statist Assoc. 2001; 96(456):1348-1360.

Fan J, Li R. Variable selection for cox proportional hazards model and frailty model. Ann Statist. 2002; 30:74-99.

Fleming, TR.; Harrington, DP. Wiley Series in Probability and Mathematical Statistics: Applied Probability and Statistics. New York: John Wiley \& Sons Inc; 1991. Counting processes and survival analysis.

Goeman JJ. L1 penalized estimation in the cox proportional hazards model. Biometrical Journal. 2010; 52(1):70-84. [PubMed: 19937997]

Huang JZ, Wu CO, Zhou L. Varying-coefficient models and basis function approximations for the analysis of repeated measurements. Biometrika. 2002; 89(1):111-128.

Hu T, Xia Y. Adaptive semi-varying coefficient model selection. Statist Sinica. 2012; 22(2):575-599.

Lin DY, Wei LJ, Ying Z. Checking the Cox model with cumulative sums of martingale-based residuals. Biometrika. 1993; 80(3):557-572.

Liu M, Lu W, Shore RE, Zeleniuch-Jacquotte A. Cox regression model with time-varying coefficients in nested case-control studies. Biostatistics. 2010; 11(4):693-706. [PubMed: 20525697]

Mallows CL. Some comments on Cp. Technometrics. 1973; 15(4):661-675.

Schwarz G. Estimating the dimension of a model. Ann Statist. 1978; 6(2):461-464.

Tian L, Zucker D, Wei LJ. On the Cox model with time-varying regression coefficients. J Amer Statist Assoc. 2005; 100(469):172-183.

Tibshirani R. Regression shrinkage and selection via the lasso. J Roy Statist Soc Ser B. 1996; 58(1): 267-288.

Tibshirani R. The lasso method for variable selection in the cox model. Statistics in Medicine. 1997; 16(4):385-395. [PubMed: 9044528]

Wang H, Xia Y. Shrinkage estimation of the varying coefficient model. J Amer Statist Assoc. 2009; 104(486):747-757.

Wang HJ, Zhu Z, Zhou J. Quantile regression in partially linear varying coefficient models. Ann Statist. 2009; 37(6B):3841C-3866.

Yan J, Huang J. Model selection for cox models with time-varying coefficients. Biometrics. 2012; 68(2):419-428. [PubMed: 22506825]

Yu Z, Lin X. Semiparametric regression with time-dependent coefficients for failure time data analysis. Statist Sinica. 2010; 20:853-869.

Zhang HH, Cheng G, Liu Y. Linear or nonlinear? automatic structure discovery for partially linear models. J Amer Statist Assoc. 2011; 106:1099-1112.

Zhang HH, Lu W. Adaptive Lasso for Cox's proportional hazards model. Biometrika. 2007; 94(3): 691-703.

Zhang W, Lee SY, Song X. Local polynomial fitting in semi-varying coefficient model. J Multivariate Anal. 2002; 82(1):166-188.

Zou H. The adaptive lasso and its oracle properties. J Amer Statist Assoc. 2006; 101(476):1418-1429.

Zucker DM, Karr AF. Nonparametric survival analysis with time-dependent covariate effects: a penalized partial likelihood approach. Ann Statist. 1990; 18(1):329-353. 

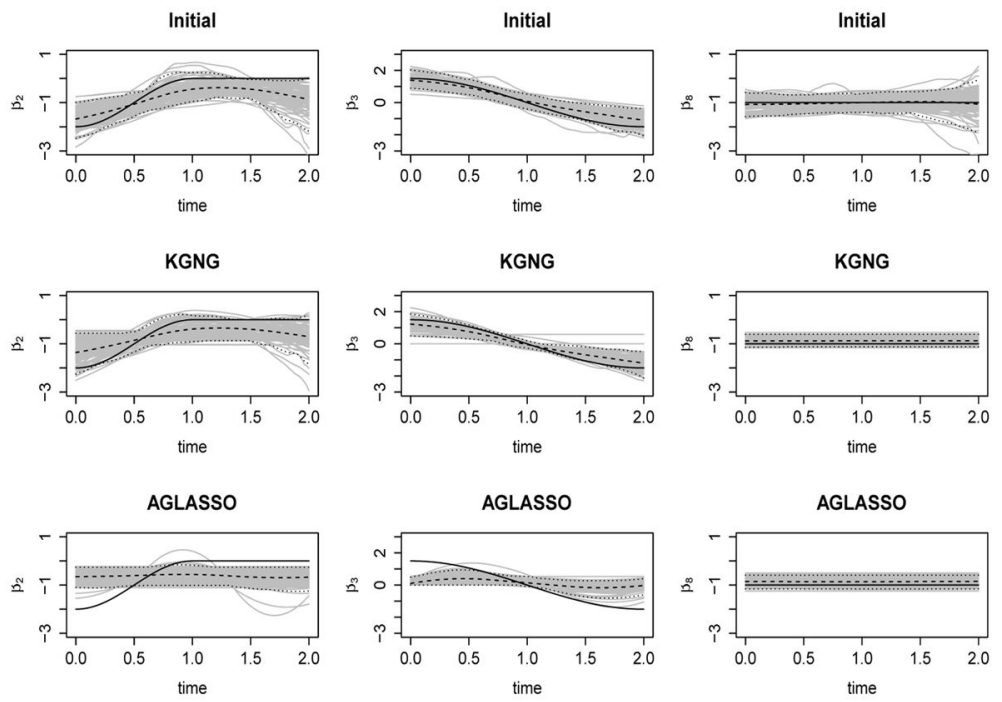

(a) $n=200$. Upper: Initial estimate. Middle: KGNG. Lower: AGLASSO.
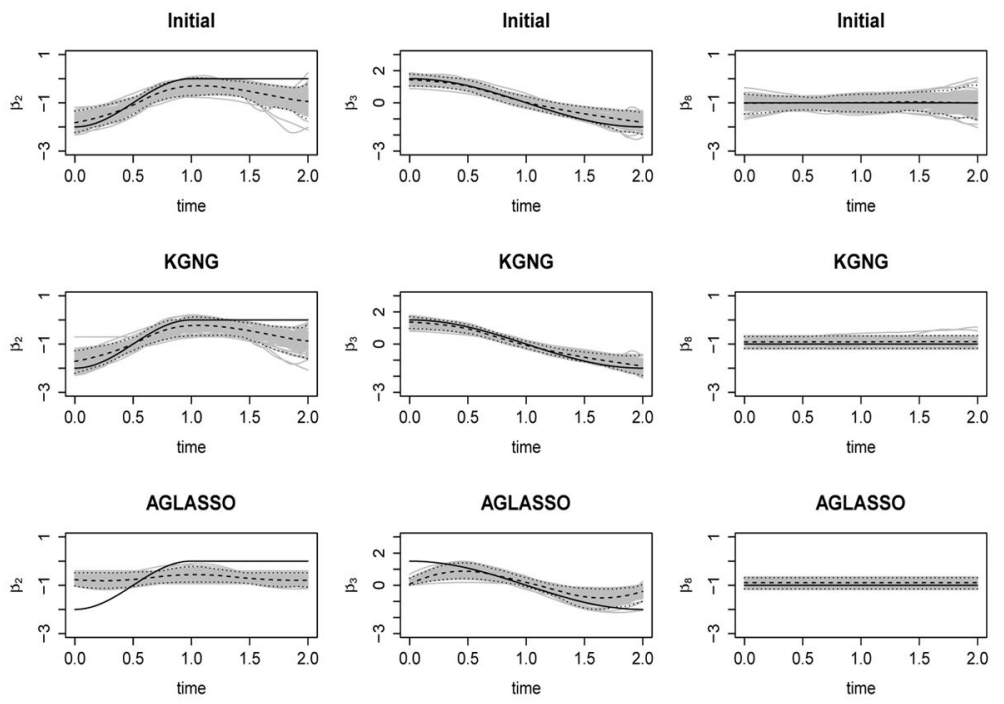

(b) $n=400$. Upper: Initial estimate. Middle: KGNG. Lower: AGLASSO.

Figure 1.

Estimated curves (gray) of the three nonzero coefficients from 100 replicates when $c_{p}=20 \%$ and $p=10$. The dark lines are the true curves. The dashed lines are the average of 100 estimates. The dotted lines are the simulation-based pointwise $95 \%$ confidence intervals. 

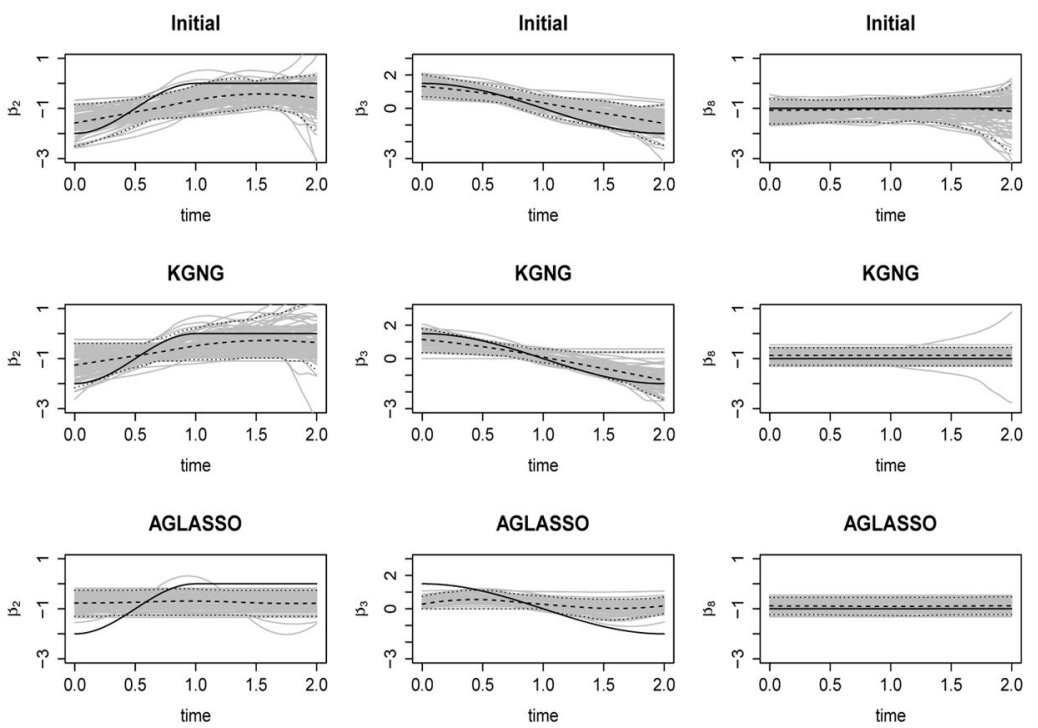

(a) $n=200$. Upper: Initial estimate. Middle: KGNG. Lower: AGLASSO.
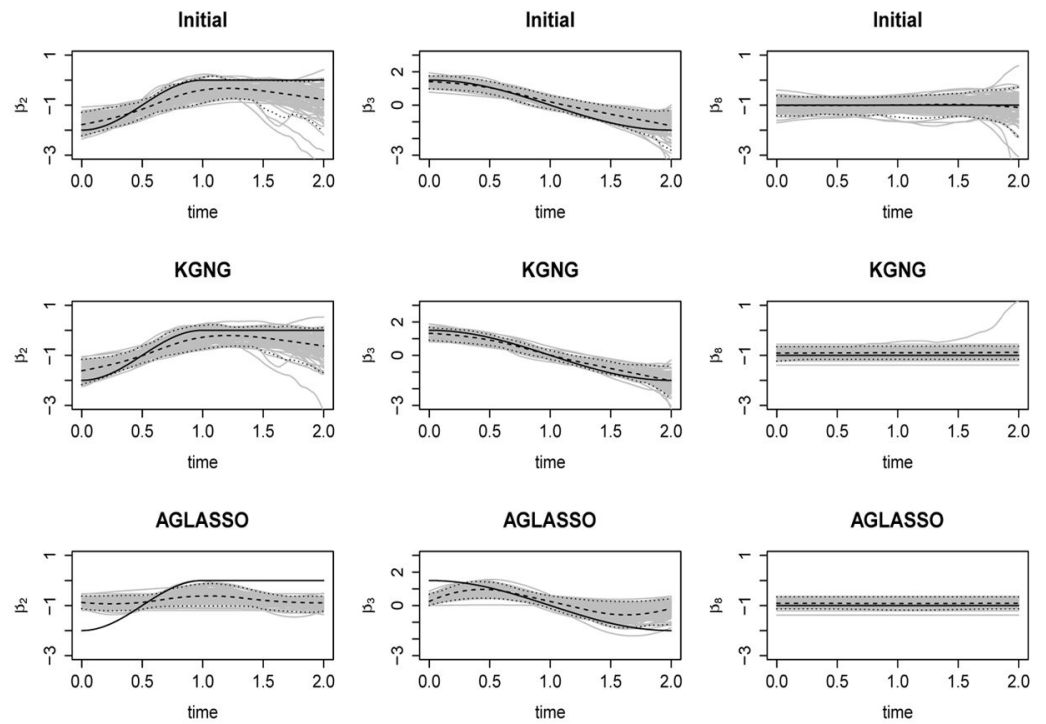

(b) $n=400$. Upper: Initial estimate. Middle: KGNG. Lower: AGLASSO.

Figure 2.

Estimated curves (gray) of the three nonzero coefficients from 100 replicates when $c_{p}=40 \%$ and $p=10$. The dark lines are the true curves. The dashed lines are the average of 100 estimates. The dotted lines are the simulation-based pointwise $95 \%$ confidence intervals. 

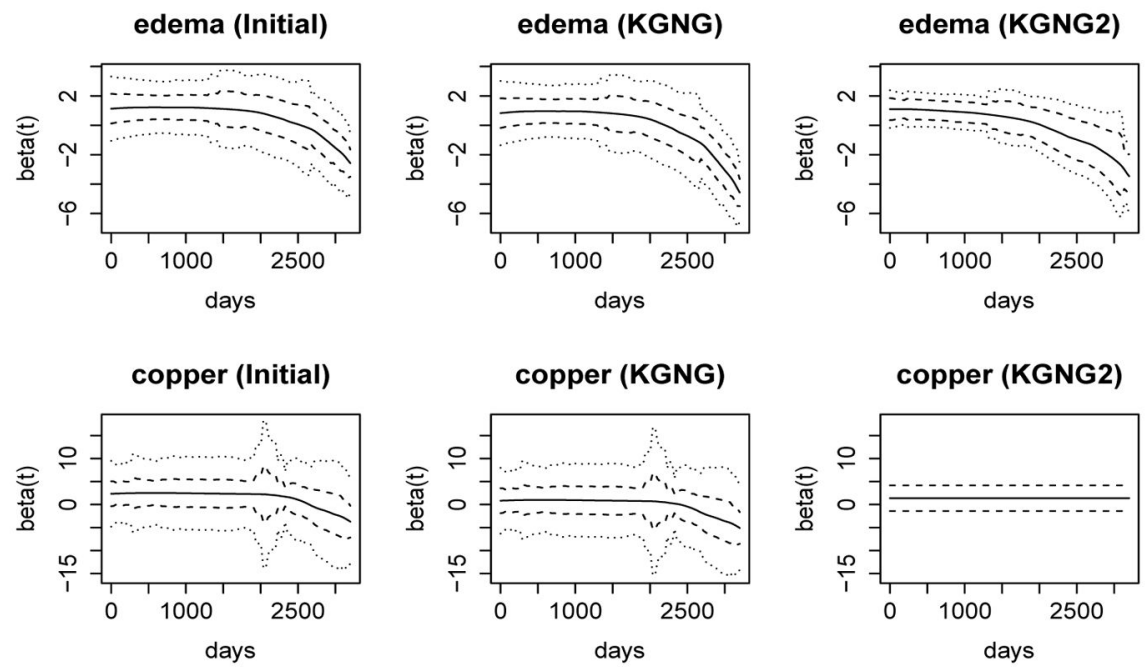

copper (KGNG2)

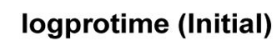

logprotime (KGNG)
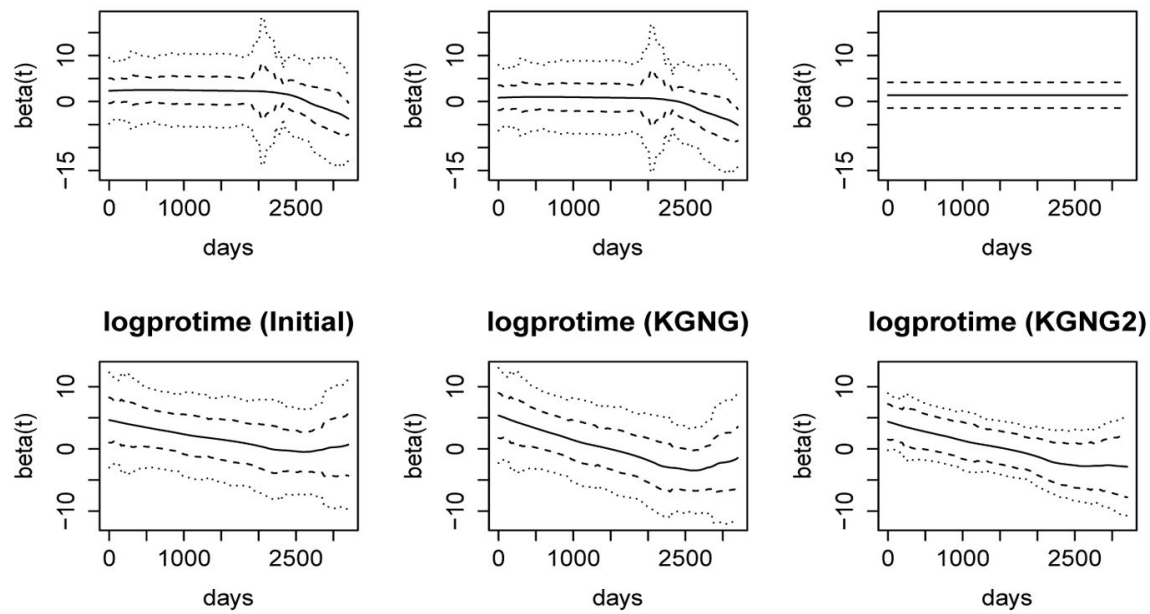

logprotime (KGNG2)

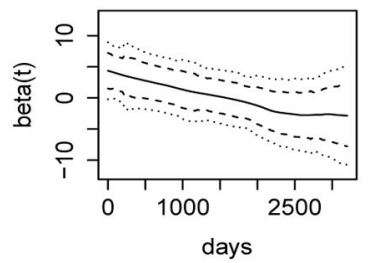

Figure 3.

Estimated coefficients for covariates: edema, copper, and logprotime. Left panel: initial estimator in KGNG; Middle panel: KGNG; Right panel: KGNG2. Solid lines: estimated curves; Dashed lines: $95 \%$ pointwise confidence intervals; Dotted lines: $95 \%$ simultaneous confidence bands. 

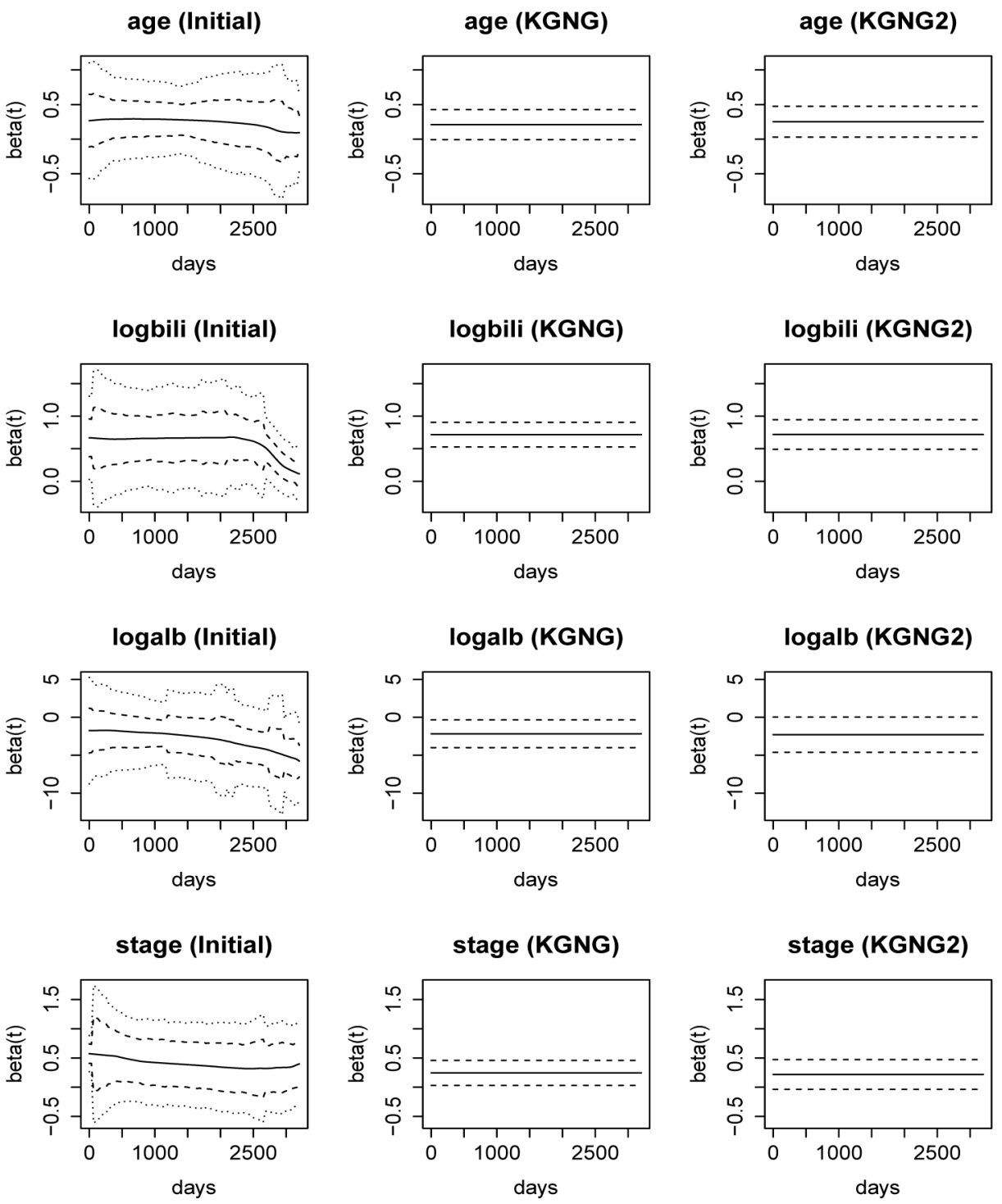

Figure 4.

Estimated coefficients for covariates: age, logbili, logalb and stage. Left panel: initial estimator in KGNG; Middle panel: KGNG; Right panel: KGNG2. Solid lines: estimated curves; Dashed lines: 95\% pointwise confidence intervals; Dotted lines: 95\% simultaneous confidence bands. 


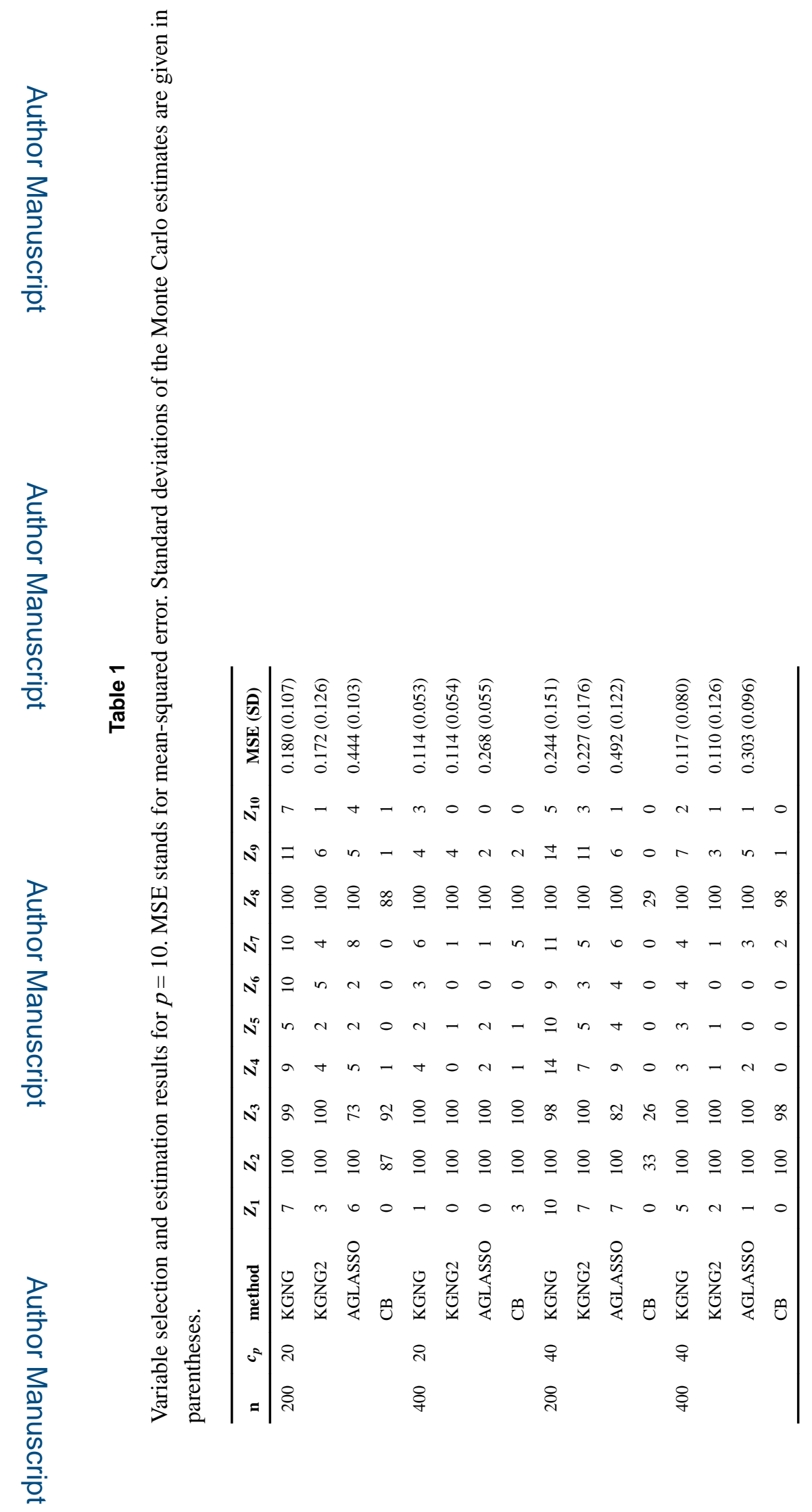

Stat Sin. Author manuscript; available in PMC 2016 August 16. 


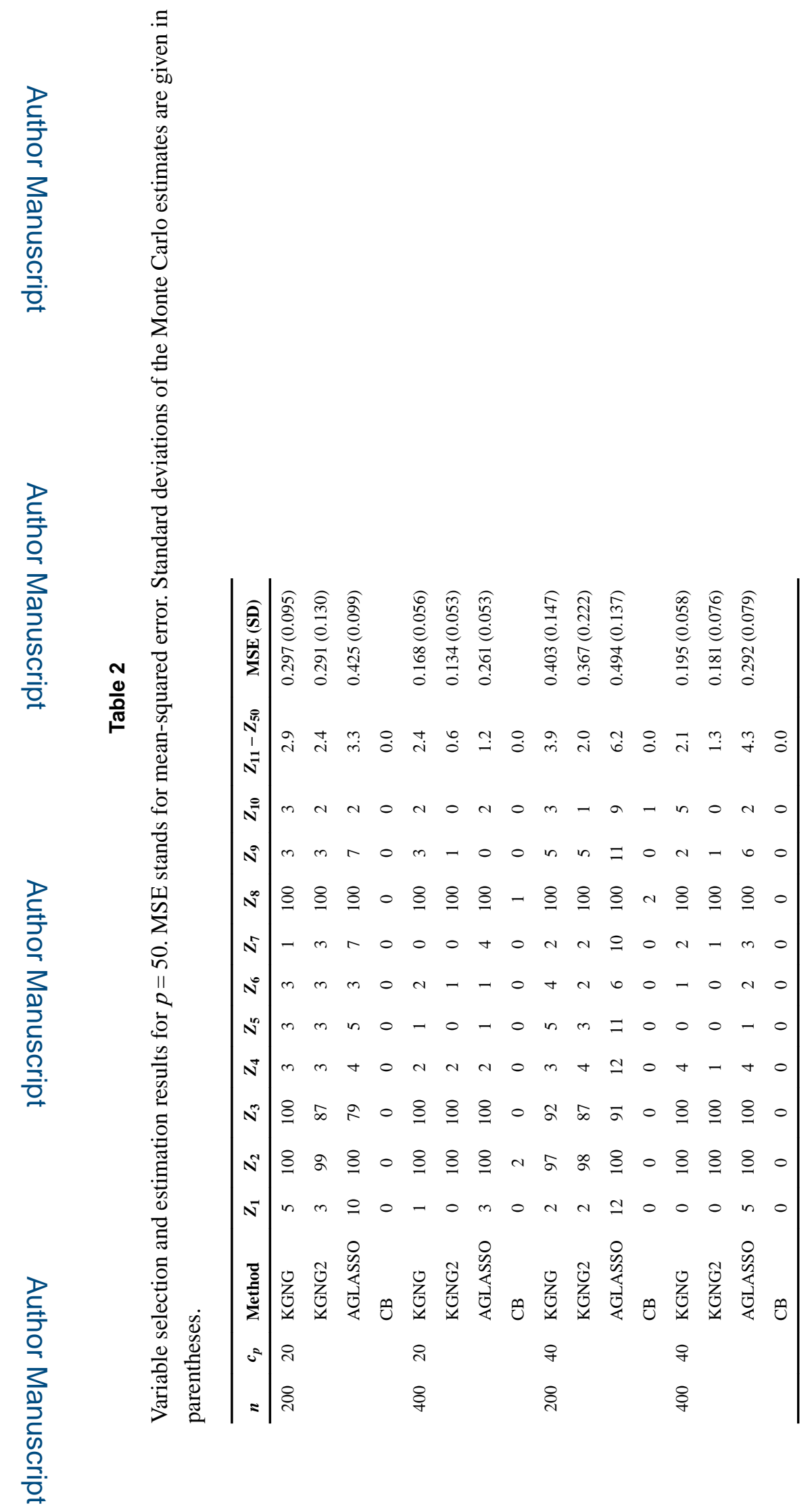

Stat Sin. Author manuscript; available in PMC 2016 August 16. 


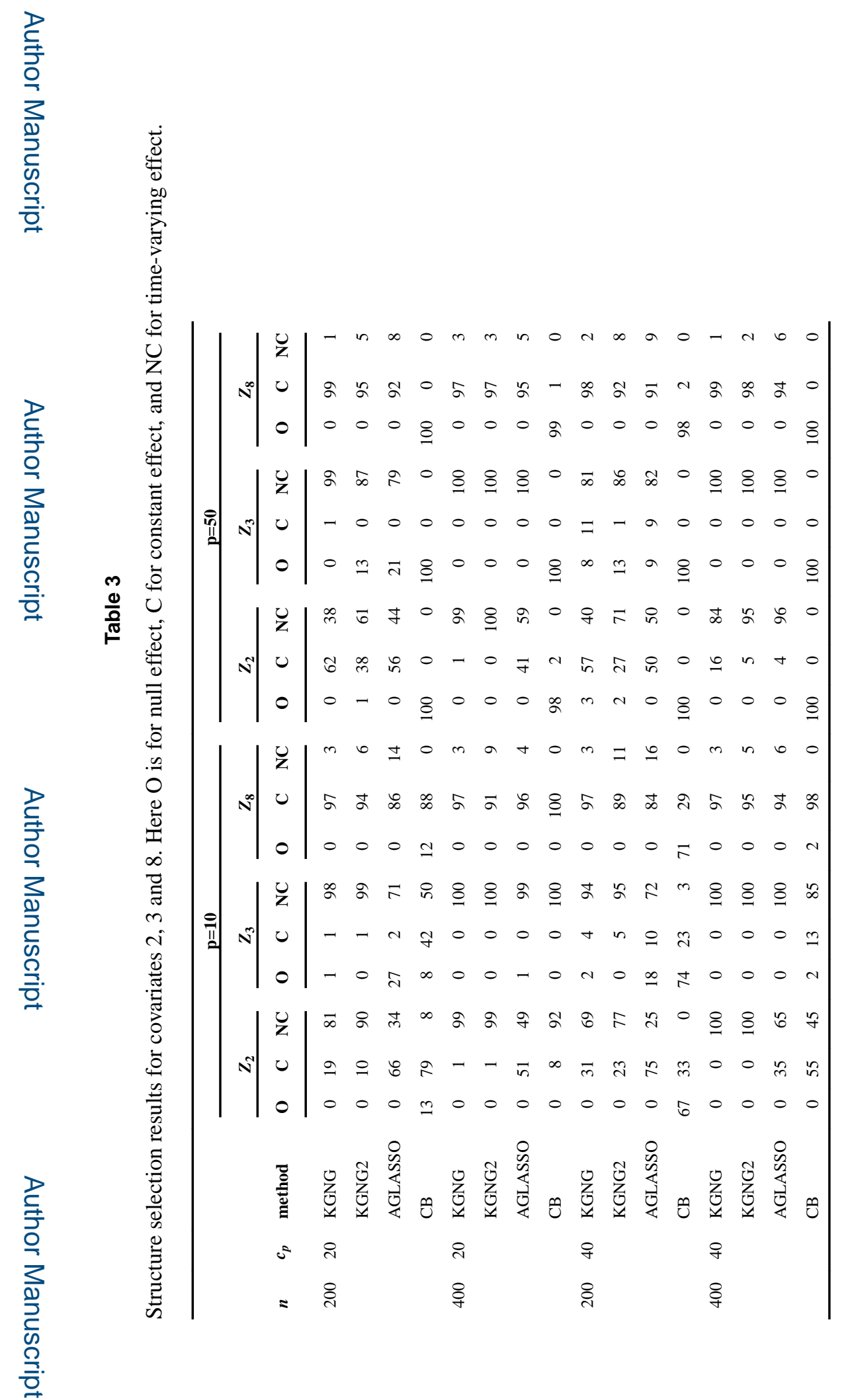




\section{을 \\ 交}

I

2

$\frac{0}{\frac{0}{0}}$

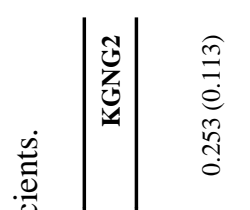

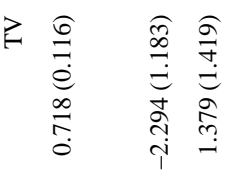

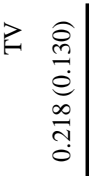

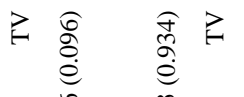

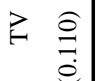

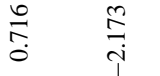

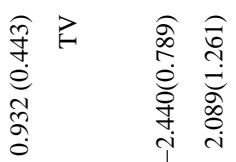

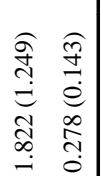

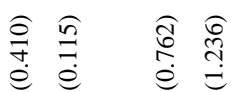

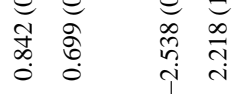

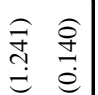

वे

苞

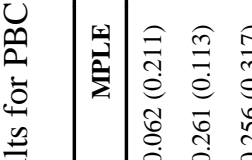

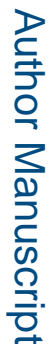

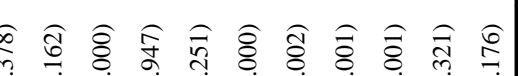

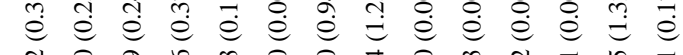

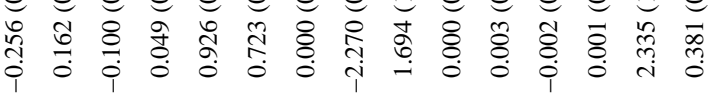

声

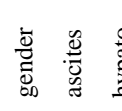

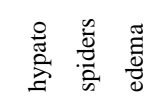

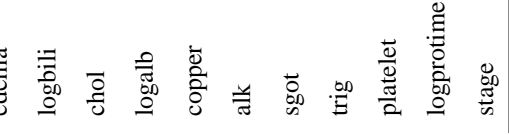

